\title{
La política criminal uruguaya en la era progresista (2005-2020): el caso de la justicia para adolescentes
}

\section{Gabriel Tenenbaum Ewig* (1)}

\section{Resumen}

El ascenso del Frente Amplio en el año 2005 a la presidencia del Uruguay generó grandes expectativas en buena parte de la sociedad uruguaya, que auguraba transformaciones sustantivas en el país. La sensibilidad de izquierdas del partido de gobierno anunciaba la incorporación del enfoque de derechos en la legislación nacional, el proceso judicial y las instituciones de ejecución de medidas, además de un amplio proceso de descriminalización y profundización en los problemas sociales y educativos. Al inicio del gobierno se realizaron cambios sustantivos, aunque no sin tensiones y frenos. Luego, paulatinamente se desarrolló una política de endurecimiento coercitivo, penal y policiaco, ante el crecimiento continuo de los principales indicadores de criminalidad (homicidios, rapiñas y hurtos). En este contexto, algunas adolescencias fueron receptoras del "giro punitivo progresista", a pesar de que la evidencia disponible sostiene que las y los adolescentes están muy distantes de explicar el delito y la criminalidad nacional. Así las cosas, el presente texto se propone problematizar la política criminal progresista para el caso del sistema de responsabilidad penal adolescente durante los gobiernos del Frente Amplio (2005 - 2020) en Uruguay. Se trata de evidenciar las "sensibilidades" que guiaron las políticas públicas criminales del partido de gobierno y plantear una discusión acerca de los dilemas de las izquierdas con respecto a la seguridad pública.

Palabras clave: justicia para adolescentes, política criminal, Uruguay.

\footnotetext{
* Universidad de la República, Montevideo, Uruguay.
} 


\section{Uruguayan criminal policy in the progressive era (2005-2020): the case of juvenile justice}

\section{Abstract}

The presidency of the Frente Amplio in 2005 generated high expectations in large part of Uruguayan society. Due to their "sensibility", a shift towards the rights approach and a process of decriminalization was expected. In the beginning, the new government made substantial changes, although not without tension and brakes. Then, perhaps surprisingly, heavy-handed policies were increased. The "adolescents" were, among others, the recipients of the punitive turn, even though they had far from explaining crime and criminality. The present research intends to problematize these subjects studying the left-wing criminal policies in the case of the special juvenile justice system during the governments of the Frente Amplio (20052020) in Uruguay. It is about showing the "sensibilities" that guided the criminal public policies of the government party and the possible explanations for this. The research realizes a descriptive study based on the triangulation of quantitative and qualitative research techniques.

Keywords: juvenile justice, criminal policies, Uruguay.

\section{Presentación}

E n el siglo XXI, acaeció un acontecimiento político sin precedentes en la sociedad uruguaya. Por primera vez en su historia, un partido político de izquierdas gobernó el país. El Frente Amplio (FA), integrado por una amplia coalición de agrupaciones políticas, algunas de las cuales cuentan con más de 100 años de recorrido ${ }^{1}$, comenzó a disputar las elecciones nacionales en 1971. A poco de su fundación, la coalición de izquierdas fue proscripta y reprimida por la dictadura (1973-1985). El partido retomó la senda electoral en 1989, aunque se presentó acéfalo² en las elecciones de1984.

En el 2004, el FA ganó su primera elección nacional y en marzo de 2005 comenzó su gestión presidencial, misma que se extendió hasta febrero

1 El Partido Socialista del Uruguay se fundó en 1910 y el Partido Comunista de Uruguay en 1920.

${ }^{2}$ Su principal líder político, Líber Seregni, aún permanecía proscripto. 
de 2020 al ganar las siguientes dos elecciones nacionales (2009 y 2014). De esta manera, una coalición de agrupaciones políticas posicionadas a la izquierda del espectro ideológico retuvo durante 15 años la presidencia de la república en las primeras dos décadas del siglo XXI.

Resulta difícil resumir lo que significó la elección del 2004 para el FA y sus votantes. Grosso modo, es posible pensar que el partido representó el cambio de una sensibilidad social (Elias, 2009; Barrán, 2004), es decir, la transformación de la estructura emotiva gobernante. Este proceso, que podemos denominar "frenteamplismo", estuvo acompañado por un sistema de creencias y cargas emocionales que entendían que se estaba rompiendo con la hegemonía de los partidos tradicionales, la dictadura cívico-militar (aunque la misma haya finalizado en 1985), el neoliberalismo, el imperialismo, entre otras ideas aglutinantes de las izquierdas que fueron combinadas con un relato moderado que le permitió llegar a un conjunto más amplio de la población sin perder su identidad como partido de cambio (Yaffé, 2005). En definitiva, el frenteamplismo representó simbólicamente una sensibilidad que venía a redistribuir los recursos económicos con base en una idea de justicia social y a ampliar el reconocimiento de derechos a diversos sectores postergados de la población (Butler; Fraser, 2016; Fraser; Honneth, 2006). Si el FA concretó o no lo que se viene diciendo, es harina de otro costal.

Cuando el FA asumió la presidencia de la república en el 2005, la modernización de la policía nacional, el sistema penitenciario de adultos, la institución rectora de ejecución de medidas judiciales para adolescentes y la invisibilidad de las violencias de género estaban entre los principales problemas públicos relativos a la seguridad. Junto con un crecimiento significativo del presupuesto destinado al Ministerio del Interior (MI), se encararon reformas importantes cuyos efectos, sin embargo, no se reflejaron en la disminución sostenida de las estadísticas delictivas. La crisis en la seguridad interna quedó patentizada en el tercer gobierno progresista (marzo 2015- febrero 2020), aunque la tendencia general del "comportamiento delictivo" haya aumentado constantemente desde la recuperación democrática - con períodos estacionarios. Entre 2017 y 
2018 los homicidios consumados aumentaron 45.8\%, las denuncias de rapiñas consumadas y tentativas se incrementaron $53.8 \%$ y las denuncias de hurtos consumadas y tentativas subieron 25.6\% (Ministerio del Interior, 2019a; 2019b). ${ }^{3}$ La tendencia se amortiguó en el 2019. Entre 2018 y 2019 los homicidios consumados disminuyeron $6 \%$, las denuncias de rapiñas consumadas y tentativas se incrementaron $2.5 \%$ y las denuncias de hurtos consumadas y tentativas se redujeron 4.3\% (Ministerio del Interior, 2020a; 2020b). ${ }^{4}$ Ante este panorama, los partidos de la oposición al gobierno comenzaron, poco a poco, a intensificar los debates acerca de la izquierda y su manejo de la política criminal. Las movilizaciones sociales, la opinión pública y los medios de comunicación colaboraron en poner sobre la agenda de discusión los dilemas de la izquierda en el manejo de la política de seguridad. Básicamente, se acusaba que el Frente Amplio trataba con benevolencia a las personas que cometían infracciones, no era capaz de imponer mano firme, orden y respeto. La crítica a la gestión de la política criminal del frenteamplismo puede resumirse en buena medida con lo que Garland (2001) denomina la criminología del otro peligroso - una perspectiva que propone una regresión hacia la demanda del castigo, abandonando cualquier intento de comprensión del delito. Se propone disuadir, castigar e incapacitar sin reparar en los costos y pone especial atención en los delitos menores como los hurtos y las rapiñas, así como en las incivilidades.

La oposición política sacó frutos del relato basado en la gestión ineficiente de la izquierda: el FA no sabe conducir con firmeza la seguridad nacional, el FA tiene temor de utilizar la mano dura para enfrentar al delito etc. Por otro lado, parte de la población uruguaya (incluido un subconjunto importante de votantes y políticos del FA) comenzó a apropiarse del relato opositor. Un estudio mostró que buena parte de la sociedad apoyaba la instrumentación

${ }^{3}$ Algunos estudios y figuras políticas señalaron que el nuevo Código de Proceso Penal de noviembre de 2017 provocó el alza de la criminalidad.

${ }^{4}$ Los Informes del Ministerio del Interior advierten que las cifras de 2019 pueden variar por motivo de subestimación a causa de retrasos en el ingreso de la información. 
de medidas de castigo más duras contra el delito, en especial contra los jóvenes (Gambeta et al., 2019). En este contexto, el frenteamplismo se encontró en una disyuntiva de extrema tensión que interpeló su "sensibilidad de izquierda" y las políticas públicas que venía implementando para mitigar el delito y el estado punitivo. Paulatinamente, como se mostrará, el FA, un partido político con una sensibilidad construida contra la represión de las instituciones del Estado, con un discurso "emancipador" de los sectores vulnerables y apegado a la clase obrera, pasó a implementar algunas medidas de seguridad provenientes de las sensibilidades de derechas.

A partir de este recorrido, el artículo problematiza la política criminal de los 15 años de gobierno del FA teniendo como base de referencia la estructura emotiva del partido. Dada la imposibilidad de abordar todas las dimensiones de la política criminal, se observa, metodológicamente hablando, el caso del sistema de responsabilidad penal adolescente uruguayo. El trabajo realiza un análisis crítico ensamblando acontecimientos decisivos que permiten delinear eventos históricos claves en la construcción de la política criminal de la coalición de izquierdas. En términos generales, la propuesta de investigación se inserta en el marco de los dilemas y discusiones generales en torno a las políticas públicas de los gobiernos progresistas latinoamericanos (Moreira, 2017; Modonesi, 2019).

Desde un diseño de investigación cualitativo, se estudia el caso del sistema de responsabilidad penal adolescente uruguayo durante los 15 años de gobierno del Frente Amplio (2005-2020). La elección se justifica a razón del sistemático posicionamiento que tienen los jóvenes, en particular los adolescentes señalados por la ley, entre los principales problemas públicos nacionales al momento de explicar el aumento de la criminalidad nacional, la alteración del orden público, los miedos y el hartazgo social acerca de la inseguridad (Tenenbaum; Viscardi, 2018).

El trabajo realiza una reconstrucción histórica de eventos de inflexión para el sistema de responsabilidad penal adolescente a través de un recorrido normativo, institucional y de políticas públicas promovidas por el poder ejecutivo entre marzo de 2005 y febrero de 2020. Este recorrido se apoya 
en el análisis de documentos secundarios, bibliografía antecedente y la realización de 14 entrevistas semiestructuradas a actores calificados del sistema de justicia para adolescentes ${ }^{5}$. Con esta propuesta, el trabajo no se propone construir una base de datos de todos los eventos relacionados con el objeto de estudio, sino destacar los eventos de inflexión, las rupturas sustantivas, que reflejan las sensibilidades y las políticas criminales de la era progresista. En otras palabras, no se enlista cada una de las propuestas políticas, los programas de acción y las intervenciones, sino que se resaltan las reformas fundamentales que significaron mojones de inflexión para el sistema de justicia adolescente. También se señalan algunas políticas públicas de seguridad nacionales que sirven para contextualizar las tendencias en las gestiones de gobierno.

A continuación, se presenta una problematización teórica dedicada a las disputas de sensibilidades en materia de política criminal. Luego, se describen cronológicamente una serie de mojos y puntos de inflexión de la política nacional en seguridad y del sistema de responsabilidad penal especial para adolescentes que muestran las orientaciones de la política criminal del FA en sus tres periodos de gobierno. El artículo finaliza con algunas reflexiones generales sobre el problema de investigación y futuras líneas de trabajo.

\section{Sensibilidad, política criminal y punitividad}

La política criminal se inscribe dentro del Estado moderno en diálogo con el derecho penal preexistente. Es la "reacción" estatal contra la "acción" de aquellas personas señaladas por la legislación. En principio, podemos

\footnotetext{
${ }^{5}$ Entre los años 2014-2015 se hicieron 10 entrevistas a operadores judiciales: cuatro jueces, tres defensores, dos peritos, un fiscal. En el año 2020 se realizaron cuatro entrevistas a operadores judiciales: un fiscal, un defensor, un mediador, un operador social. El objeto de realizar estas entrevistas fue el de relevar información sobre cambios normativos y de política criminal y judicial, así como conocer el funcionamiento del sistema de justicia para adolescentes en la era progresista. Los operadores judiciales son informantes clave (unidades de registro), mas no unidad de análisis. Por esa razón, y a los efectos de construir una lectura fluida, el autor elige elaborar un texto sin citas. Sin embargo, el desarrollo cronológico de los acontecimientos de inflexión que reflejan las sensibilidades y las políticas criminales de la era progresista está basado, entre otras fuentes, en esta técnica de investigación.
} 
suponer que la finalidad de la política criminal es mitigar el delito, aunque bien sabemos que éste no es propósito homogéneo. Existen mecanismos de priorización de la persecución penal y desigualdad en la distribución de las vigilancias y los castigos.

La política criminal, así como la legislación, traslucen la sensibilidad de personas de influencia, partidos políticos y sectores sociales movilizadores de intereses con incidencia temporal en el aparato estatal. Las decisiones acerca del tipo, la intensidad y la preferencia sobre una u otra política de prevención, vigilancia, control y/o castigo están condicionadas a una o más estructuras emotivas y morales, y hasta existenciales, además de la experiencia y el derrotero de conocimientos que existen en el campo de estudio y sus disciplinas.

Lo que aquí conocemos como sensibilidad tiene un vasto recorrido aplicado a los estudios sobre el delito y el crimen - desde Durkheim (1999) con los conceptos de conciencia moral y sentimientos colectivos, Garland (2001; 2006) con "artefactos culturales" y la "cultura del control", hasta Spierenburg (2013) con la sensibilidad eliasiana. Para comprender esto, veamos una tipología ${ }^{6}$ compuesta por dos bloques polares de sensibilidades que se mantienen vigentes hasta nuestros días.

Por una parte, en el bloque punitivo se halla la ideología de la defensa social y el realismo de derecha. El punto de partida de la ideología de la defensa social es una concepción ideal, teleológica y moral de lo que tiene que ser una sociedad. Filipo Gramatica y Marc Ancel fueron los principales exponentes de la Escuela de la Defensa Social europea. Creada en la década de 1940, la "Escuela" tuvo una fuerte influencia en América Latina. Promovió un abordaje donde la protección social se opone radicalmente al hecho delictivo (del Olmo, 2010), dejando a las personas perpetradoras de delitos en la categoría expulsora y meritocrática de los antisociales no "dignos" de la protección estatal. Un abordaje de este tipo le entrega

${ }^{6}$ La tipología es una delineación general aproximada y no un monolito con ajuste perfecto. Tiene el propósito de teorizar y orientar la interpretación del material empírico. 
sentido a la función de la cárcel como espacio y arquitectura destinada a la exclusión, el apartamiento y la represión de los antisociales. Con Giorgio Agamben (2003), podríamos decir que los antisociales son aquellas personas reducidas a meros cuerpos, jurídicamente "indeterminados" y desprotegidos. Para Alessandro Baratta (2013, p. 42), la defensa social "corresponde a una ideología caracterizada por una concepción abstracta y ahistórica de sociedad entendida como una totalidad de valores e intereses." La crítica de Baratta plantea una separación epistémica, directa y concreta, con aquellas lecturas criminológicas liberales que responsabilizan al individuo aislado y dividen la realidad social entre nosotros y los otros.

En el bloque punitivo también se reconoce al realismo de derecha. Pegoraro (2001, p. 154), quien afirma que Platt y Takagi (1981) fueron los primeros en identificar a los "realistas de derecha", entiende que la corriente representa un conjunto variado de pensamientos identificados bajo el "continuum de ley y orden: orden social-estado soberano-política penal y en considerar a la delincuencia común como el enemigo principal de la vida social". Una de las vertientes más importantes de esta sensibilidad es la teoría de las "ventanas rotas" y de la "desorganización social". La teoría de las "ventanas rotas" data, según la historia oficial, de un artículo de James Wilson y George Kelling publicado en 1982 en la revista estadounidense The Atlantic ${ }^{7}$. Sin embargo, los primeros antecedentes se reconocen a finales de la década de 1960 con los experimentos del psicólogo Philip Zambardo y en los programas de patrullaje policial del Estado de New Jersey. Asimismo, quizás sea posible remontarse a la teoría ecológica y culturalista de la Escuela de Chicago, en particular a los estudios sobre la delincuencia juvenil en comunidades urbanas desordenadas de los años treinta y cuarenta del siglo XX.

7 The Atlantic, James Wilson e George Kelling, "Broken Windows. The police and neighborhood safety". March 1982 issue. En: https://www.theatlantic.com/magazine/ archive/1982/03/broken-windows/304465/. Consultado el 9 de abril de 2020. 
La característica esencial de la teoría de las ventanas rotas es la primacía del orden por sobre todas las cosas y más allá de cómo se logre (Wilson; Kelling, 2016). Wilson sostenía que el mantenimiento del orden debía ser ponderado por sobre la impartición de justicia y el bienestar económico en la explicación del delito (Aller, 2011). Esta aseveración tiene consecuencias directas sobre, por ejemplo, la distribución del presupuesto de un Estado en tanto priorizara el fortalecimiento del control y la vigilancia policiaca en el espacio público, ${ }^{8}$ en detrimento de programas de reinserción social, contra la pobreza, la desigualdad etc. El ejemplo paradigmático del realismo de derecha es la política de "tolerancia cero" durante la alcaldía de Rudolph Giuliani en Nueva York, entre los años 1994 y 2002. Al respecto, Loïc Wacquant (2004, p. 32) sostiene que este tipo de política criminal utiliza la "retórica militar de la 'guerra' al crimen y de la 'reconquista' del espacio público, que asimila a los delincuentes (reales o imaginarios), los sin techo, los mendigos y otros marginales a invasores extranjeros". Este bloque de sensibilidad suele inscribirse en lo que Paternain (2012) llama "hegemonía conservadora".

La ideología de la defensa social y el realismo de derecha son sensibilidades que representan fehacientemente el malestar social acoplado de manera cuasi perfecta a las fuerzas seductoras del punitivismo. Promueven el populismo penal: "el deseo de ver sufrir a los delincuentes y vengar a las víctimas, la preocupación explícita por expresar la indignación pública, el reclamo de que debe hacerse justicia, de que la autoridad debe ser defendida" (Garland, 2001, p. 91). Lo que debe quedar claro es que esta estructura emocional no se levanta a causa de la criminalidad, sino por el malestar que produce el desvelo sistemático de lo que implica lograr y mantener cierto "orden único" y la distribución intacta de los privilegios materiales y simbólicos.

Por otra parte, el bloque crítico apuntala la esfera de lo social y pone al descubierto las relaciones de poder y los intereses en juego. Aquí

${ }_{8}^{8}$ Algunos ejemplos: mantenimiento de calles, veredas, alumbrado, ornato público, discrecionalidad alrededor de la alternación del orden público (ley de faltas) etcétera. 
están las sensibilidades que devienen de perspectivas como la teoría del etiquetamiento, la filosofía foucaultiana, la criminología crítica y el realismo de izquierda. No todas ellas son propositivas, mas sí todas parecen decodificar, a su modo, la simulada neutralidad de la política criminal. Aunque hay diferencias dentro y entre las perspectivas, algunas de las cuales no son simples matices, es posible sostener que ellas conforman un bloque crítico en relación con el bloque punitivo y que en todas se hallan hondas preocupaciones sociales. Entienden que la política criminal trasluce intereses particulares y arbitrarios, que su configuración responde a procesos estructurales económicos, sociales y culturales relacionados con el poder y la dominación. Asistimos a un sistema penal estrechamente vinculado con la clase social. Este sistema distribuye selectiva y desigualmente el castigo. Por esta razón, desde esta sensibilidad se entiende que la desviación social no es una cualidad del acto que la persona realiza, sino una consecuencia de la aplicación de la norma y el castigo que otros aplican (Becker, 2014). La desviación es una respuesta frente al control social, es una reacción desarticulada, pero con contenido sociopolítico, contra la desintegración y las privaciones económicas y de derechos. A grandes rasgos, esta sensibilidad tiene dos propuestas de cambio: (i) la erradicación de las clases sociales a través de un cambio radical en las condiciones de producción de la sociedad capitalista; (ii) avances, aunque sean moderados, en justicia social y reconocimiento de derechos de los sectores postergados, silenciados y olvidados. Los precursores de este bloque tipológico, orientado a desneutralizar las enseñanzas de la escuela liberal clásica y del positivismo alrededor de la defensa del orden social, son, quizás, Willem Bonger, Georg Rusche y Otto Kirchheimer en las primeras décadas del siglo XX. También se debe mencionar la influencia de la criminología crítica italiana y de Michel Foucault en la obra de, por ejemplo, Rosa del Olmo, Lola Aniyar de Castro, Roberto Bergalli, Juan Pegoraro, entre otros destacados especialistas latinoamericanos. En la actualidad, otros académicos como Loïc Wacquant, David Garland y Didier Fassin arremeten contra las políticas punitivas de corte neoliberal que caracterizan a los Estados modernos occidentales. 
Para cerrar, es importante delimitar lo que se entiende por punitividad, dado que es uno de los factores clave para diferenciar a los bloques presentados. La punitividad comprende "Ios niveles de dolor o sufrimiento producidos por el sistema penal” (Sozzo, 2016, p. 10). Las características de las políticas punitivas, siguiendo a Didier Fassin (2018, p. 11), son "leyes más duras, asociando la automaticidad y la agravación de penas y de prácticas más inflexibles de la institución penal...en un contexto de desigualdades y violencias". En sintonía, para Garland (2001) lo punitivo es un estilo de "ley y orden" donde el poder soberano se expande desmesuradamente y reafirma su segregacionismo. Por otro lado, para Michael Tonry, los países con mayor probabilidad de aplicar políticas punitivas se caracterizan por: concentrar la autoridad en el gobierno (quizás en pocas personas) y politizar el crimen; alta capacidad de influencia de reconocidos medios de comunicación sensacionalistas, víctimas y opinión pública sobre las autoridades; bajo nivel de confianza y credibilidad en el sistema legal; y alto nivel de inequidad social, baja solidaridad social y escasa inversión en políticas de bienestar social (Trajtenberg, 2012). Por otra parte, especialistas como Matthews (2005) han criticado a los teóricos de la punitividad por falta de rigurosidad en la evidencia que utilizan y por el carácter fenomenológico del concepto, en tanto son las expectativas normativas y las sensibilidades públicas, lo que se valora apropiado y excesivo, lo que explica lo que entendemos por punitividad. La punitividad, entonces, está históricamente situada y atada a las estructuras emocionales; lo que representa un problema a los historiadores del castigo que buscan responder si nuestros tiempos son más o menos pacíficos que otras épocas.

Sin ingresar en los debates epistémicos y metodológicos sobre la delimitación de la punitividad, para los fines de este trabajo es posible indicar que la ideología de la defensa social y el realismo de derecha maximizarán la punitividad del Estado, mientras que una sensibilidad sociocrítica la minimizará. Empero, como la realidad es más compleja, 
cabe preguntarse si pueden presentarse "momentos punitivos" ${ }^{\text {en la }}$ sensibilidad sociocrítica, en la era progresista. De otra manera, ¿es posible pensar en una sensibilidad de izquierda punitiva? Este problema se indagará a continuación para el caso uruguayo.

\section{Trazos de la política criminal en los gobiernos del Frente Amplio}

En el primer quinquenio de gobierno, bajo la administración de Tabaré Vázquez, la medida que marcó la tendencia de la política criminal del FA fue la aprobación de la Ley n¹7.897 de "Libertad Provisional y Anticipada" (Ley de Humanización Carcelaria) de setiembre de 2005. La norma estuvo dirigida a adultos internados en las penitenciarías nacionales con el objetivo de otorgar libertades provisionales y anticipadas, prisiones domiciliarias y otros beneficios a personas de edad avanzada, primarios etc. La norma refleja bastante bien la sensibilidad de izquierda y fue aprobada a los pocos de meses de iniciar el nuevo gobierno.

Posiblemente, las resistencias más firmes a la transformación que buscaba implementar el FA vinieron del Ministerio del Interior, de la policía. En ese periodo, la cartera ministerial tuvo 3 ministros y 2 viceministros: Jorge Díaz-Juan Faroppa entre el 01/03/2005 y el 08/03/2007; Daisy Tourné-Ricardo Bernal entre el 08/03/2007 y el 05/06/2009; y Jorge BruniRicardo Bernal entre el 05/06/2009 y el 01/03/2010 (Vernazza, 2015). De alguna manera, en este primer ciclo de gobierno, al FA le costó mucho desplazar a la vieja cultura policial. Para Paternain (2017, p. 163), el FA asumió "la conducción de la seguridad sin diagnósticos claros y sin hojas de

\footnotetext{
${ }^{9}$ Didier Fassin conoce por "momento punitivo" al incremento de la cantidad y severidad de infracciones a la ley, incluso cuando la actividad delictiva disminuye. El momento punitivo es la emergencia del castigo como problema y no, a pesar de que así suele ser planteado, como solución del delito. Para autores como Garland, Wacquant y Fassin el giro regresivo se produce entre las décadas de 1960 y 1980 en los países occidentales (especialmente en los países desarrollados y, en concreto, en Estados Unidos, Reino Unido y Francia).
} 
ruta precisas para enfrentar los retos de un ámbito desconocido, riesgoso y resistente a cualquier impulso transformador". El país estaba saliendo de la crisis de 2002 y la prioridad la tenía la indigencia y la pobreza. De alguna manera, por contexto y falta de expertise, el FA "compró" el diagnóstico de la criminalización de la pobreza al creer que solucionando las necesidades básicas insatisfechas mitigaría directa e indefectiblemente el delito. La pobreza bajó sostenidamente (Colafranceschi et al., 2013), pero las denuncias de rapiñas (Ministerio del Interior, 2019a, 2019b) ${ }^{\mathbf{1 0}}$ y la tasa de prisionización no dejaron de aumentar (CPP, 2020). La oposición política y determinados sectores sociales llevaron adelante una intensa crítica a la primera gestión de la seguridad del FA. Sustentaban que el gobierno de izquierda era incapaz de implementar medidas duras, de impacto, contra el delito. Se construyó un relato uniforme alrededor de la idea de "estado de guerra", con enemigos sociales llamados delincuentes a los que el FA favorecía con sus políticas sociales. La crítica se transformó en dilema y fue calando lentamente en la opinión pública y en algunos dirigentes y sectores políticos de la coalición de izquierdas.

En materia de adolescentes, el desafío más importante que enfrentó la primera gestión del FA fue instrumentar el enfoque de derechos con la reciente aprobación del Código de la Niñez y la Adolescencia (CNA) en setiembre de 2004. Junto a ello, debía brindar una solución definitiva a los innumerables escollos y violaciones de derechos que se venían manifestando en el Instituto Técnico de Rehabilitación Juvenil (INTERJ), la institución estatal de ejecución de medidas judiciales para adolescentes. Sin embargo, el primer gobierno del FA experimentó graves problemas con las fugas de los centros de internación: "la probabilidad de fuga de los hogares del INAU creció desde niveles cercanos al 10 por ciento en 1997 hasta niveles del

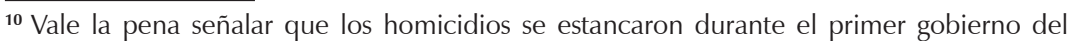
Frente Amplio para luego aumentar constantemente. Por otra parte, las denuncias de hurtos muestran un comportamiento llamativo. El hurto venía en aumento desde el año 1998 y en el año 2005, con la asunción del Frente Amplio, la tendencia se frena y cae unos años hasta que nuevamente retoma la senda creciente. 
entorno del 40 por ciento en 2010" (Munyo, 2012, p. 8). Las violencias, las condiciones indignas de vida y los suicidios eran otros problemas graves que enfrentó la institucionalidad (INDDHH, 2018). Recién a finales de 2009, al término del mandato de Vázquez, se tomaron medidas de raíz con la sustitución del INTERJ por el Sistema de Ejecución de Medidas para Jóvenes en Infracción (SEMEJI). En paralelo, en el primer ciclo de gobierno del FA, aumentaron las detenciones policiales, los procesos judiciales y las sentencias contra los adolescentes (Tenenbaum; Viscardi, en prensa). ${ }^{11}$

Durante el segundo gobierno del FA, bajo la presidencia de José Mujica (2010-2015), se acordaron y sancionaron leyes que "endurecieron" la política criminal. Un mojón clave fue el fortalecimiento de la seguridad y la vigilancia de las penitenciarías, además de la necesaria reforma organizacional, edilicia y de servicios de salud y reinserción social. Ello se hizo con la aprobación de la Ley n 18.667 de julio de 2010 sobre el "Sistema Penitenciario Nacional". Según el informe 2019 del Comisionado Parlamentario Penitenciario (2020), esta reforma penitenciaria, basada en las normas y estándares de derechos humanos, fue posible por la presión que ejerció la visita del relator de Naciones Unidas, Manfred Nowak, en el 2009. Pero el punto de inflexión durante la presidencia de Mujica fue el Consenso Interpartidario sobre Seguridad Pública (en adelante, Consenso) del 10 de agosto de 2010. El acuerdo implicó 14 reuniones entre el poder ejecutivo y los representantes de todos los partidos políticos. Buscó construir una política de Estado en materia de seguridad y, como correlato, mermar el antagonismo con el ministro del Interior y el partido de gobierno. Los acuerdos alcanzados concretaron la Ley n 18.717 de diciembre de 2010 sobre el "Personal Militar" que dispuso la responsabilidad de la guardia perimetral y el control de acceso de las penitenciarías nacionales al ejército nacional. La medida fue un hito histórico ya que, tras el régimen militar

11 En todo el país, los principales delitos cometidos por adolescentes son las rapiñas y los hurtos. Entre 2009 y 2019, el promedio país de rapiñas y hurtos de adolescentes sentenciados por la justicia fue $75,4 \%$. Mientras en Montevideo predomina la rapiña, en el interior lo hace el hurto (Tenenbaum; Viscardi, en prensa). 
que gobernó el país entre 1973 y 1985, se trató de mantener alejadas a las Fuerzas Armadas de la seguridad interna. El Consenso también ocasionó un aumento significativo del presupuesto dirigido al Ministerio del Interior entre los años 2005 y $2015 .^{12}$

El Consenso se focalizó en varias acciones para enfrentar el "delito adolescente" que, en esos años, más precisamente entre 2011 y 2013, alcanzó los datos más altos del siglo XXI. En el año 2009, los adolescentes sentenciados por la justicia fueron 1559. En el 2011 se alcanzó el pico más alto de la década con 1947 sentencias contra adolescentes. Luego, las cifras comenzaron a bajar constantemente. En el 2014 se calcularon 1513 sentencias y en el 2017 se reportaron 1048 sentencias contra adolescentes (Tenenbaum; Viscardi, en prensa). Vale la pena notar que las niñeces vulneradas en la crisis del 2002 llegaron a ser adolescentes en los primeros años del segundo gobierno del FA. Casualmente o no, la población atendida en el sistema penal especial para adolescentes llegó a la cima en el segundo ciclo del FA.

EI SEMEJI, creado a finales de 2009, siguió presentando los problemas de su antecesor INETRJ y fue reemplazado en julio de 2011 por el Sistema de Responsabilidad Penal Adolescente (SIRPA) a impulso de la Ley $n^{\circ} 18771$ para el perfeccionamiento del sistema de responsabilidad penal juvenil. Ello ya había sido acordado en el 2010 por el Consenso entre los partidos políticos: "la creación de un Instituto de Rehabilitación de los Adolescentes en conflicto con la ley penal como organismo especializado (acuerdo 3.16.1) [...] con el más alto grado de autonomía técnica" (Comisión Interpartidaria de Seguridad Pública, 2010, p. 10). Otro de los acuerdos alcanzados fue el número 3.19.6 respecto al estudio de "modificaciones legislativas que

\footnotetext{
${ }^{12}$ En 2005, el presupuesto del Ministerio del Interior era de 4.882.819.501 pesos uruguayos (200.115.553,4 dólares americanos con un tipo de cambio de 24,4 pesos por dólar) y en 2015 era de 21.643.105.403 pesos uruguayos (792.787.743,7 dólares americanos con un tipo de cambio de 27,3 pesos por dólar). Fuente: UYCheck (2019) 4 chequeos del debate presidencial: educación, seguridad y economía. En: http://uycheck.com/4-chequeos-deldebate/. Consultado el 15 de noviembre de 2019.
} 
aseguren la aplicación de medidas socioeducativas o privativas de libertad a todas las infracciones cometidas por los adolescentes a la ley penal" (Comisión Interpartidaria de Seguridad Pública, 2010, p. 10). Este fue el puntapié para la sanción de tres normas que endurecieron el sistema especial de responsabilidad penal adolescente: (i) La Ley $\mathrm{n}^{\circ} 18.777$ del 15 de julio de 2011 criminalizó la tentativa y la complicidad del delito de hurto y la sancionó con medidas socioeducativas no privativas de libertad. La norma amplió el plazo de las medidas cautelares privativas de la libertad de 60 a 90 días para infracciones gravísimas; (ii) la Ley $n^{\circ} 18.778$, también del 15 de julio de 2011, aprobó la creación del "Registro Nacional de Antecedentes Judiciales de Adolescentes en Conflicto con la Ley Penal". La norma modificó el artículo 222 del CNA para permitir al juez la posibilidad de conservar los antecedentes de los adolescentes, en determinados delitos (rapiña, violación, homicidio intencional, copamiento y secuestro), a efectos de ser utilizados cuando los involucrados cumplan la mayoría de edad y cometan delito doloso o ultraintencional; (iii) la Ley $\mathrm{n}^{\circ} 19.055$ de enero de 2013 sancionó la internación cautelar preceptiva de los adolescentes entre 15 y 17 años, por supuestas infracciones gravísimas ${ }^{13}$, hasta el dictado de la sentencia definitiva. La legislación definió que la duración de la pena por infracciones gravísimas no puede ser inferior a los 12 meses. Díaz y Fernández (2017) mostraron que la bancada del FA en la Cámara de Representantes apoyó disciplinadamente el proyecto de ley, a pesar de la oposición de expertos y organizaciones de la sociedad civil. La Institución Nacional de Derechos Humanos y Defensoría del Pueblo señaló que la normativa genera diferencias inentendibles entre adultos y adolescentes porque en los adultos la prisión preventiva no es preceptiva en ningún caso. Además, la ley contradice las recomendaciones internacionales acerca de

${ }^{13}$ Las infracciones gravísimas son: homicidio intencional agravado, lesiones gravísimas, violación, rapiña, privación de libertad agravada, secuestro y otra acción u omisión que castigue la Ley Penal o las leyes especiales...También son infracciones gravísimas, pero no están comprendidas en este punto: la extorción, el tráfico de estupefacientes, la tentativa y la complicidad en el homicidio intencional agravado, violación, rapiña y secuestro. 
los principios de excepcionalidad, brevedad y especificidad de la pena privativa de libertad (INDDHH, 2018). Para Gandelman y Munyo (2015) estas medidas frenaron significativamente la reincidencia delictiva.

La aprobación de la triada normativa estuvo acompañada por movilizaciones políticas y sociales de gran envergadura, que buscaron intensificar la capacidad punitiva del Estado sobre las adolescencias. Uno de estos movimientos fue impulsado en el 2011 por la agrupación política "Vamos Uruguay" del Partido Colorado que, junto con el apoyo de algunos sectores del Partido Nacional, formaron la Comisión por la Seguridad para Vivir en Paz-Yo firmo. La comisión reunió la cantidad de firmas suficientes - igual o más de 250.000 firmas de electores (10\% de los habilitados para votar) - para plebiscitar la disminución de la edad de imputabilidad penal de 18 a 16 años. También se propuso no destruir los registros antecedentes de los adolescentes judicializados a fin de utilizarlos, llegado el caso, en la Justicia Penal de adultos - propuesta que, como se leyó en el apartado anterior, fue legislada en el 2011. La reforma de la edad de imputabilidad no era novedosa. La ideología de la defensa social y el realismo de derecha venían intentando este cambio desde 1985. Desde la última recuperación del régimen de gobierno democrático en 1985 hasta el 2020 se presentaron "16 intentos legales (14 Proyectos de Ley, una iniciativa plebiscitaria y una propuesta por comisión parlamentaria) para cambiar el umbral etario penalmente punible" (Tenenbaum, 2011, p. 127). Finalmente, el plebiscito se concretó en octubre de 2014 y el resultado no modificó la legislación, aunque sí reflejó un país polarizado con el $46.8 \%$ del electorado a favor de reducir la edad de imputabilidad penal.

En el 2012, en medio de la movilizaciones pro-reducción de la edad de imputabilidad, el segundo gobierno del FA elaboró el documento Estrategias por la vida y la convivencia (Presidencia de la República, 2012). Entre otras cosas, el documento mencionó, vagamente, la necesidad de modificar el CNA en lo "que rige para los menores infractores", lo que se concretó en el 2013. Pero el apuntalamiento del documento se dirigió 
hacia el reconocimiento de que la política social estatal no era suficiente para enfrentar el delito. En este marco, propuso llevar adelante una política de "acupuntura urbana" para "reconstruir la trama urbana y ubicar al vecindario y al barrio como un lugar para vivir y compartir y no como un espacio ganado por la inseguridad, la falta de control y límites" (p. 17). Dicha aseveración, que bien podría ajustarse a la teoría de las ventanas rotas, se plasmó más tarde con la puesta en marcha de grandes operativos policiales (operativos de "saturación"). Esta política fue acompañada por la sanción, en agosto de 2013, de la Ley n 19120 sobre "Faltas, conservación y cuidado de los espacios públicos" (Ley de Faltas) que buscaba instalar la primacía del orden por sobre todas las cosas y judicializar "incivilidades".

El tercer ciclo de gobierno de las primeras "quince primaveras" frenteamplistas, durante el segundo mandato de Tabaré Vázquez, continuó lo que se venía haciendo en el ciclo anterior. Mantuvo al ministro del Interior y profundizó los operativos de "saturación" que pasaron a llamarse "Mirador". A diferencia de su antecesor, "Mirador" tuvo una focalización directa en las "organizaciones" criminales dedicadas al tráfico de drogas, la "recuperación" de territorios cooptados por el delito y el restablecimiento de servicios públicos. Entre diciembre de 2017 y agosto de 2019 se realizaron 29 operativos que arrojaron 616 allanamientos y 303 personas detenidas en todo el país (Tenenbaum, 2019). Los operativos fueron, básicamente, despliegues policiacos a gran escala donde participaron los cuerpos de choque y las unidades policiales especializadas en drogas ilegales e inteligencia ${ }^{14}$. En algunos casos, también participaron funcionarios de las intendencias, de las empresas públicas del Estado (agua y luz) y de la Dirección General de Impositiva. Si bien todavía no existe ningún estudio de evaluación del impacto de los operativos "Mirador", una revisión exploratoria de los decomisos da cuenta de que, en buena parte de los casos, los operativos estuvieron lejos de dar un golpe a la estructura delictiva, pero cumplieron

${ }^{14}$ La cantidad de funcionarios policiales varía por operativo, pero, para tener una idea, se movilizan desde 70 hasta 150 efectivos. Se utiliza "alta" tecnología: armas de grueso calibre, drones, helicópteros, transporte blindado etc. 
con una parte de la sociedad que reclamaba respuestas directas y punitivas. Otra de las propuestas del tercer ciclo de gobierno del FA fue el Programa de Alta Dedicación Operativa (PADO) del año 2016. Básicamente, el programa se propone patrullajes policiales a partir de evidencia empírica sobre las áreas con mayor concentración de denuncias delictivas. Las primeras evaluaciones mostraron resultados positivos, aunque tímidos, en términos de disuasión del crimen (Chainey et al., 2017; AGEV, 2016). Luego, el efecto del patrullaje por circuitos fijos ocasionó el corrimiento del delito a otros espacios dejando sin efecto el impacto del programa.

En el sistema de justicia para adolescentes, se creó una nueva institucionalidad en la ejecución de medidas judiciales. En el año 2015 se creó el Instituto Nacional de Inclusión Social Adolescente (INISA) con la aprobación de la Ley $n^{\circ}$ 19.367. El INISA es un órgano estatal descentralizado - ya no desconcentrado - separado del INAU que logró, a diferencia de sus antecedentes, brindar mejores servicios básicos, contener las fugas, bajar la conflictividad en los centros de internación, profesionalizar las propuestas socioeducativas y fortalecer la institución de ejecución de medidas judiciales para adolescentes. Los datos del INISA sobre la población atendida muestran que, entre los años 2014 y 2019, los adolescentes privados de libertad disminuyeron $49.7 \%$ y los ingresos de adolescentes con medidas en libertad ${ }^{15}$ se redujeron 36\%. En diciembre de 2020, último dato consultado, en el INISA había 321 (65\%) adolescentes privados de la libertad y 171 (35\%) adolescentes con medidas en libertad. A pesar de la disminución de la cantidad de personas atendidas en la institución, la justicia no utiliza la privación de la libertad como medida de último recurso (Tenenbaum; Viscardi, en prensa). Quizás la disminución de la población atendida sea el mayor logro de la administración INISA, aunque los nuevos ingresos no dependen completamente del quehacer de la institución. Otro

${ }^{15}$ Como en otros trabajos, el autor insiste en la utilización del concepto "medidas en libertad", en sustitución de "medidas alternativas a la privación de libertad", con la finalidad de otorgar la posición de privilegio, de primer recurso, que deben tener este tipo de medidas de reinserción social frente a las medidas privativas de la libertad. 
avance ocurrió en el 2018, con la creación e instrumentación del "modelo de intervención de sanciones no privativas de libertad para adolescentes en Uruguay". Pero poco se sabe acerca de su aplicación.

Un mojón histórico para el sistema de justicia en este tercer gobierno fue la aprobación del nuevo Código del Proceso Penal (CPP). La ley $n^{\circ}$ 19.293 del 19 de diciembre de 2014 creó el CPP16, que entró en vigor en noviembre de 2017. La nueva legislación impulsó la aprobación de la Ley n ${ }^{\circ}$ 19.551 del 18 de octubre de 2017, que modificó varios artículos del CNA para armonizar la legislación de adolescentes con la de los adultos en el pasaje del proceso inquisitivo al régimen acusatorio, adversarial y público. Armonización que no tuvo en cuenta la dureza con la que se castiga a las rapiñas de adolescentes a diferencia de los adultos, al menos en lo que se refiere a la duración mínima de la pena privativa de la libertad.

\section{A modo de cierre}

Si fuera necesario delinear los primeros 15 años de gobierno del Frente Amplio en la historia del Uruguay, se podría decir que, grosso modo, la política criminal ha sido hibrida tomando en cuenta el esquema propuesto de los bloques de sensibilidad. Estructuras emotivas dispares convivieron y se disputaron el dominio de la política pública. En los primeros dos o tres años predominó la sensibilidad de izquierda en el gobierno del FA. Luego vinieron las contradicciones y el desapego con las bases emocionales de la izquierda. La falta de diagnósticos y evidencia científica, de ideas y creatividad, parece explicar la filtración de ideas, emociones y abordajes de la sensibilidad de derecha en la búsqueda desesperada por encontrar soluciones urgentes a problemas urgentes.

${ }^{16}$ Las modificaciones al nuevo Código del Proceso Penal son: Ley $n^{\circ} 19.436$ del 23 de setiembre de 2016; Ley $n^{\circ} 19.446$ del 28 de octubre de 2016; Ley $n^{\circ} 19.544$ del 20 de octubre de 2017; Ley $n^{\circ} 19.549$ del 6 de noviembre de 2017. Los sucesivos cambios hablan de disputas corporativas en el sistema de justicia, de la crítica política y de ajustes de funcionamiento. 
El primer ciclo de gobierno intentó ser fiel a una idea de sensibilidad de izquierda anclada en el fortalecimiento del Estado de Bienestar. No obstante, las intenciones no fueron acompañadas por la aplicación de un programa de seguridad planificado, sustentado en fundamentos teóricos y empíricos que hace décadas venía desarrollando la criminología crítica, el realismo de izquierda, la teoría de etiquetamiento, entre otras corrientes de pensamiento que podrían ser afines al partido de gobierno. Por ingenuidad, convencimiento, factibilidad y/o el contexto posterior a la crisis de 2002, la apuesta estuvo en la política social. Ello no fue suficiente.

En el segundo gobierno del FA, la normativa penal contra los adolescentes señalados por la ley se endureció por lo que mostraban los guarismos, pero también por la demanda popular de punitivismo, la presión de los partidos de la oposición y hasta de ciertos sectores del propio FA. De ahí en más, la sensibilidad y el discurso de izquierda pregonado por décadas se fue combinando con posiciones inadvertidas antes del 2005. El FA se acercó a los abordajes de la teoría del enemigo, del sujeto peligroso, de la defensa social, del desorden etc. Aunque algunos sectores frenteamplistas y de la sociedad civil mostraron su desacuerdo con este giro punitivo, la reacción tardó y nunca lograron articularse y contragolpear a la sensibilidad de derecha en la interna del partido. Llamó la atención la facilidad y la rapidez con la que se retornó a las prácticas reactivas de la ideología de la defensa social y el realismo de derecha para mitigar la delincuencia; prácticas que, desde su origen, el FA se encargó de condenar sistemáticamente.

El partido de gobierno fue reposicionando su política criminal a ensayo y error, lo fue haciendo con una prótesis en su cintura que alertaba los movimientos de una opinión pública con suficientes razones de hartazgo. Así fue dejando a un lado el dominio de la sensibilidad de izquierda en la política pública criminal, para mantener una identidad hibrida intercalada en su discurso y la acción. En los últimos años de gobierno, el recetario de soluciones "insecticidas de rápida acción" (como el PADO, "Mirador" etc.) apareció como los cantos hechizantes de las sirenas de Homero. 
El progresismo uruguayo, como sucedió en otros países de la región, mostró que las debilidades de su política criminal fueron intercambiadas irreflexivamente por el discurso y la acción de sensibilidades que tensionan y hasta antagonizan con su identidad de izquierda. La misión de mitigar el delito sin detenerse en el cómo, acarreó remplazos emocionales sin cuestionamiento. Lo diferente se confundió con lo peligroso, la obsesión por "estar más seguros" socavó las divergencias de las estructuras emocionales de la izquierda y la derecha. El bloqueo de la diferencia fue vaciando los fundamentos del FA acerca de cómo una sensibilidad de izquierda debería orientar la política criminal.

Por último, resulta necesario continuar estudiando la relación entre política criminal y sensibilidad de izquierda para la era del progresismo latinoamericano que finalizó. ¿Por qué? Porque marcó un proceso histórico singular en la región que dejó contradicciones y aprendizajes. Además, en términos experimentales, puede que sea, teóricamente, un caso de contraste interesante con respecto a regímenes anteriores y posteriores.

Gabriel Tenenbaum Ewig es Doctor en Ciencia Social con Especialidad en Sociología por El Colegio de México, docente e investigador del Departamento de Sociología de la Facultad de Ciencias Sociales de la Universidad de la República, Uruguay.

$\bowtie$ gabriel.tenenbaum@cienciassociales.edu.uy

\section{Referencias}

1. AGAMBEN, Giorgio. Homo Sacer. El poder soberano y la nuda vida. Valencia: Pre-textos, 2003.

2. AGEV. Evaluación de diseño, implementación y desempeño (DID) programa de alta dedicación operativa. Montevideo: Oficina de Planeamiento y Presupuesto, 2016.

3. ALLER, Germán. Paradigmas de la criminología contemporánea. Revista de Derecho Penal y Criminología, v. 3, n. 5, p. 173-198, 2011.

4. BARATTA, Alessandro. Criminología crítica y crítica del derecho penal. Ciudad de México: Siglo XXI, 2013. 
5. BARRÁN, José P. Historia de la sensibilidad en el Uruguay. Montevideo: Banda Oriental, 2004.

6. BECKER, Howard. Outsiders. Hacia una sociología de la desviación. Buenos Aires: Siglo XXI, 2014.

7. BUTLER, Judith; FRASER, Nancy. ¿Redistribución o reconocimiento? Un debate entre marxismo y feminismo. Madrid: New Left/Traficantes de sueños, 2016.

8. CHAINEY, Spencer; VENERI, Federico; SERRANO, Rodrigo . Evaluando el impacto del Programa de Alta Dedicación Operativa (PADO) en la reducción de robos violentos en Montevideo, Uruguay. In: BONOMI, Eduardo et al. ¿Cómo evitar el delito urbano? El Programa de Alta Dedicación Operativa en la Nueva Policía Uruguaya. Montevideo: Ministerio del Interior/Banco Interamericano de Desarrollo, 2017. p. 155-180.

9. COLAFRANCESCHI, Marco; VIGORITO, Andrea; FAILACHE, Elisa. Desigualdad multidimensional y dinámica de la pobreza en Uruguay en los años recientes. Montevideo: PNUD, 2013.

10. COMISIÓN INTERPARTIDARIA DE SEGURIDAD PÚBLICA. Documento de consenso. Disponible en: https://www.minterior.gub.uy/images/stories/ documento de_consenso.pdf

11. COMISIONADO PARLAMENTARIO PENITENCIARIO. Informe 2019. Montevideo: Parlamento del Uruguay, 2020. Disponible en: https://parlamento. gub.uy/cpp

12. DEL OlMO, Rosa. América Latina y su criminología. Ciudad de México: Siglo XXI, 2010.

13. DÍAZ, Daniel; FERNÁNDEZ, Martín. Constitucionalidad y derecho de defensa. Los adolescentes frente a la Ley 19.055. In: ABELLA, Rosana; FESSLER, Daniel (comp.). El retorno del "estado peligroso". Montevideo: Casa Bertolt Brecht, 2017. p. 127-138.

14. DURKHEIM, Emilio. Dos leyes de la evolución penal. Delito y Sociedad, v. 8, n. 13, p. 71-90, 1999.

15. ELIAS, Norbert. El proceso de la civilización. Investigaciones sociogenéticas y psicogenéticas. Madrid: Fondo de Cultura Económica, 2009.

16. FASSIN, Didier. Castigar. Buenos Aires: Adriana Hidalgo, 2018.

17. FRASER, Nancy; HONNETH, Axel. ¿Redistribución o reconocimiento? Un debate político-filosófico. Madrid: Ediciones Morata, 2006.

18. GAMBETA, Victoria; MUSTO, Clara; TRAJTENBERG, Nico; VIGNA, Ana. Actitudes hacia el castigo penal: ¿qué tan punitivos somos los uruguayos? In: FILARDO, Verónica (coord.). El Uruguay desde la Sociología XVII. Montevideo: Universidad de la República, 2019. p. 201-226. 
19. GANDELMAN, Néstor; MUNYO, Ignacio. Juvenile incarceration and crime after release: evidence from a Harsher law. In: MARCH FORUM: WORKSHOP ON ECONOMICS OF CRIME, 2015, Montevideo. Anales [...]. Montevideo: Ridge, 2015.

20. GARLAND, David. Castigo y sociedad moderna. Ciudad de México: Siglo XXI, 2006.

21. GARLAND, David. La cultura del control. Crimen y orden social en la sociedad contemporánea. Barcelona: Gedisa, 2001.

22. INDDHH - INSTITUTO NACIONAL DE DERECHOS HUMANOS Y DEFENSORÍA DEL PUEBLO. Libertad, seguridad y derechos humanos. In: ASAMBLEA NACIONAL DE DERECHOS HUMANOS: NUEVAS LEYES, ¿MÁS DERECHOS?, VII, 2018, Ciudad de Paysandú. Anales [...]. Montevideo: INDDHH, 2018.

23. MATTHEWS, Roger. The myth of punitiveness. Theoretical Criminology, v. 9, n. 2, p. 175-201, 2005. https://doi.org/10.1177\%2F1362480605051639

24. MINISTERIO DEL INTERIOR. Homicidios del $1^{\circ}$ de enero al 31 de diciembre (2018-2019). Montevideo: División de estadísticas y análisis estratégico Observatorio Nacional sobre Violencia y Criminalidad, 2020a.

25. MINISTERIO DEL INTERIOR. Denuncias de rapiña y hurto del $1^{\circ}$ de enero al 31 de diciembre (2018-2019). Montevideo: División de estadísticas y análisis estratégico - Observatorio Nacional sobre Violencia y Criminalidad, 2020b.

26. MINISTERIO DEL INTERIOR. Homicidios del $1^{\circ}$ de enero al 31 de diciembre (2017-2018). Montevideo: División de estadísticas y análisis estratégico Observatorio Nacional sobre Violencia y Criminalidad, 2019a.

27. MINISTERIO DEL INTERIOR. Denuncias de rapiña y hurto del $1^{\circ}$ de enero al 31 de diciembre (2017-2018). Montevideo: División de estadísticas y análisis estratégico - Observatorio Nacional sobre Violencia y Criminalidad, 2019b.

28. MODONESI, Massimo. El progresismo latinoamericano: un debate de época. In: GAUDICHAUD, Franck et al. (coords.). Los gobiernos progresistas latinoamericanos del siglo XXI. Ensayos de interpretación histórica. Ciudad de México: Universidad Nacional Autónoma de México, 2019. p. 181-229.

29. MOREIRA, Constanza. El largo ciclo del progresismo latinoamericano y su freno: los cambios políticos en América Latina de la última década (20032015). Revista Brasileira de Ciências Sociais, v. 32, n. 93, p. 1-28, 2017.

30. MUNYO, Ignacio. Los dilemas de la delincuencia juvenil en Uruguay. Montevideo: CERES, 2012.

31. PATERNAIN, Rafael. Políticas de policía y gobiernos de izquierda. El caso de Uruguay. Delito y Sociedad, v. 44, n. 26, p. 161-200, 2017.

32. PATERNAIN, Rafael. La hegemonía conservadora en el campo de la seguridad. Una interpretación del caso uruguayo. Crítica Contemporánea. Revista de Teoría Política, n. 2, p. 83-100, 2012. 
33. PEGORARO, Juan. Derecha criminológica, neoliberalismo y política penal. Delito y Sociedad, v. 1, n. 15-16, p. 141-160, 2001. https://doi.org/10.14409/dys. $\underline{\mathrm{v} 1 \mathrm{i} 15 / 16.5473}$

34. PLATT, Tony; TAKAGI, Paul (ed.). Critical criminology. Crime and social justice. Londres: The MacMillan Press, 1981.

35. PRESIDENCIA DE LA REPÚBLICA. Estrategias por la vida y la convivencia. Montevideo: Presidencia de la República de Uruguay, 2012. Disponible en: https:// medios.presidencia.gub.uy/jm_portal/2012/noticias/NO_E582/Estrategia.pdf

36. SOZZO, Máximo. Postneoliberalismo y penalidad en América del Sur. Buenos Aires: CLACSO, 2016.

37. SPIERENBURG, Pieter. Violence and punishment. Civilizing the body trough time. Cambridge: Polity Press, 2013.

38. TENENBAUM, Gabriel. La brújula. Breve rodeo sobre la distribución desigual de la persecución delictiva durante los gobiernos del Frente Amplio. Hemisferio Izquierdo, n. 33, 2019. Disponible en: https://www.hemisferioizquierdo.uy

39. TENENBAUM, Gabriel. La discusión legislativa de la edad de imputabilidad en los anales de la recuperación democrática. Cualquier semejanza con la actualidad NO es pura coincidencia. Revista de Ciencias Sociales, n. 24, v. 28, p. 127-147. 2011.

40. TENENBAUM, Gabriel; VISCARDI, Nilia. Avances, retrocesos y problemas en la justicia para adolescentes uruguaya del siglo XXI. In: ALVARADO, Arturo; TENENBAUM, Gabriel (coords.). Los desafíos de la justicia para adolescentes en América Latina. Avances y retrocesos en el enfoque de derechos humanos. Ciudad de México: El Colegio de México, en prensa.

41. TENENBAUM, Gabriel; VISCARDI. Nilia (coords.). Juventudes y violencias en América Latina. Sobre los dispositivos de coacción en el siglo XXI. Montevideo: Universidad de la República, 2018.

42. TRAJTENBERG, Nicolás. Sistema de justicia penal. Explicaciones de la punitividad. Revista de Ciencias Sociales, v. 25, n. 31, p. 59-78, 2012.

43. VERNAZZA, Lucía. El dilema de las políticas de seguridad en los gobiernos de izquierda. Uruguay 2005 - 2014. (Tesis de Maestría) Facultad Latinoamericana de Ciencias Sociales, Buenos Aires, 2015.

44. WACQUANT, Loic. Las cárceles de la miseria. Buenos Aires: Manantial, 2004.

45. WILSON, James; KELLING, George. Ventanas rotas. La policía y la seguridad en los barrios. Delito y Sociedad, v. 1, n. 15/16, p. 69-79, 2016.

46. YAFFÉ, Jaime. Al centro y adentro. La renovación de la izquierda y el triunfo del Frente Amplio en Uruguay. Montevideo: Linardi y Risso, 2005.

Recibido: 27 ago. 2021. Aprobado: 17 nov. 2021. 
